Proyecciones

Vol. $11 \mathrm{~N}^{\circ}$ 2, pp.143-151 Diciembre 1992

Universidad Católica del Norte

Antofagasta - Chile

\title{
ON THE CONTINUITY OF LIMIT CAPACITY OF CENTRAL CANTOR SETS
}

\author{
EDUARDO MUÑOZ M.* \\ JAIME VERA $V$.
}

Universidad Católica del Norte, Antofagsta, Chile.

\begin{abstract}
A metric topology is defined on the family of central Cantor sets (not necessarily proportionals). In this topology the Hausdorff dimension as well as the limit capacity depend continuously on the central Cantor set. An example of continuous arcs with CONSTANT limit capacity is given.
\end{abstract}

* The author acknowledges kind IMPA hospitality during the period this work was done. Work partially supported by UCANORTE.

** The author acknowledges kind IMPA hospitality during the period this work was done. Work partially supported by FONDECYT Grant 90-0901, UCANORTE and IMPA-CNPq. 


\section{Basic Concepts and Result.}

We begin with the definitions of Hausdorff dimension and limit capacity. Let $U$ be a subset of the metric space $(X, \rho)$, the diameter of $U$ is $|U|=$ $\sup \{\rho(x, y): x, y \in U\}$. If $A \subset \cup_{i \in I} U_{i}$ and $0 \leq|U| \leq \delta$ for every $i$ then we say $\left(U_{i}\right)_{i \in I}$ is a $\delta$-covering of $A$.

For every $A \subset X, s>0$ and $\delta>0$ the $(s, \delta)$-Hausdorff measure of $A$ is defined by:

$$
\mathcal{H}_{\delta}^{s}(A)=\inf \left\{\sum_{i=1}^{\infty}\left|U_{i}\right|^{s}:\left(U_{i}\right)_{i \in \mathbb{N}} \quad \text { is a countable } \delta \text { - covering of } A\right\}
$$

From the definition above it follows that if $\delta_{1}<\delta_{2}$ then $\mathcal{H}_{\delta_{2}}^{s}(A) \leq \mathcal{H}_{\delta_{1}}^{s}(A)$, so the function $\delta \longrightarrow \mathcal{H}_{\delta}^{s}(A)$ is not increasing and we can define the s-Hausdorff measure for the set $A$ as:

$$
\mathcal{H}^{s}(A)=\sup _{\delta>0} \mathcal{H}_{\delta}^{s}(A)=\lim _{\delta \rightarrow 0^{+}} \mathcal{H}_{\delta}^{s}(A)
$$

It is easy to see that there is a unique number $\alpha$ such that

$$
\mathcal{H}^{s}(A)=\left\{\begin{aligned}
+\infty & \text { if } s<\alpha \\
0 & \text { if } s>\alpha .
\end{aligned}\right.
$$

The graphic of $s \longrightarrow \mathcal{H}^{s}(A)$ is shown in Figure 1 .

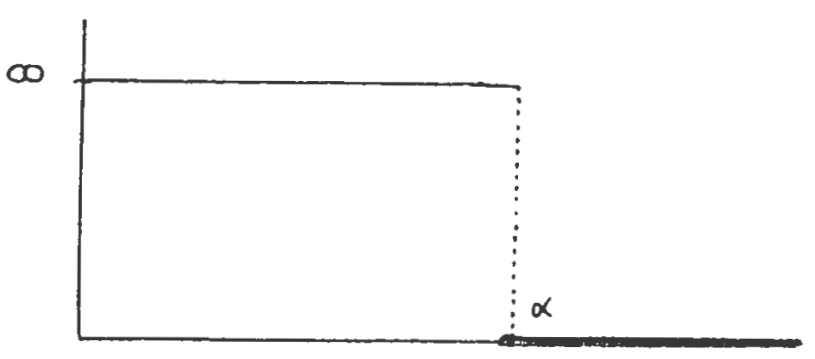

Figure 1 
This number $\alpha$ is defined as the Hausdorff dimension of $A$ :

$$
H D(A)=\sup \left\{s>0: \mathcal{H}^{s}(A)=+\infty\right\}=\inf \left\{s>0: \mathcal{H}^{s}(A)=0\right\}=\alpha .
$$

Let $K$ be a compact subset of the metric space $(X, \rho)$. Given $\epsilon>0$, we denote by $n(\epsilon, K)$ the minimun number of open balls of radius $\epsilon$ needed to cover $K$. The limit capacity of $K, d(K)$, is defined by:

$$
d(K)=\limsup _{\epsilon \rightarrow 0} \frac{\ln n(\epsilon, K)}{-\ln \epsilon} .
$$

Denote by $S^{+}$the set of sequence $\eta=\left\{\eta_{i}\right\}_{i=1}^{\infty}$ such that $\eta_{i}>0$ and $\sum_{i=1}^{\infty} \eta_{i}=1$. For each sequence $\eta \in S^{+}$we associate a central Cantor set in the following way: Let $K_{0}=[0,1]$ and suppose we have defined the sets $K_{1}, \ldots, K_{n-1}$, then $K_{n}$ is defined deleting in each connected component of $K_{n-1}$ the central open interval of length $2^{-(n-1)} \eta_{n}$, so $K_{n}$ is the union of $2^{n}$ disjoint closed intervals of length $2^{-n}\left(1-\sum_{i=1}^{n} \eta_{i}\right)=2^{-n}\left(\sum_{i=n+1}^{\infty} \eta_{i}\right)$. The central Cantor set associated to the sequence $\eta$ is the zero Lebesgue measure Cantor set $K(\eta)=\cap_{n \in N} K_{n}$.

We let denote $\mathcal{K}$ as the class of central Cantor sets defined above. We have the following

Theorem 1. There is a metric topology defined on $\mathcal{K}$ such that the apllications $K \longrightarrow d(K)$ and $K \longrightarrow H D(K)$ are continuous.

\section{Topology on $\mathcal{K}$}

Here we let us endowe the class of central Cantor sets $\mathcal{K}$ with a metric topology. In this topology the Hausdorff dimension and the limit capacity are continuous functions of the Cantor sets.

Let $K=\cap_{n=0}^{\infty} K_{n}, \epsilon>0$ and $I_{n}$ any connected component of $K_{n}$, then the $\epsilon$-neighbourhood of $K$ is defined as

$$
B(K, \epsilon)=\left\{\tilde{K}=\bigcap_{n=0}^{\infty} \tilde{K}_{n} \mid \exists 0<\tilde{\epsilon}<\epsilon \text { such that } 2^{-\tilde{\epsilon} n}<\frac{\ell\left(I_{n}\right)}{\ell\left(\tilde{I}_{n}\right)}<2^{\tilde{\varepsilon} n}, n \in \mathbb{N}\right\}
$$

where $\ell\left(I_{n}\right)$ denotes the length of $I_{n}$. Note that if $\tilde{K} \in B(K, \epsilon)$, then there is $\epsilon_{1}<\epsilon$ such that $B\left(\tilde{K}, \epsilon_{1}\right) \subset B(K, \epsilon)$. In fact, let $\epsilon_{1}<\epsilon-\tilde{\epsilon}$ where $0<\tilde{\epsilon}<\epsilon$ is 
such that

$$
2^{-i n}<\frac{\ell\left(I_{n}\right)}{\ell\left(\tilde{I}_{n}\right)}<2^{\tilde{\epsilon} n}, n \in \mathbb{N}
$$

Take $\bar{K} \in B\left(\tilde{K}, \epsilon_{1}\right)$, then there is $0<\tilde{\epsilon}_{1}<\epsilon_{1}$ such that

$$
2^{-\tilde{\epsilon}_{1} n}<\frac{\ell\left(\tilde{I}_{n}\right)}{\ell\left(\bar{I}_{n}\right)}<2^{\tilde{\varepsilon}_{1} n}
$$

Combining the last two inequalities we obtain

$$
2^{-\left(\tilde{\epsilon}+\tilde{\epsilon}_{1}\right) n}<\frac{\ell\left(I_{n}\right)}{\ell\left(\tilde{I}_{n}\right)}<2^{\left(\tilde{\epsilon}+\tilde{\epsilon}_{1}\right) n}
$$

and since $\epsilon_{1}+\tilde{\epsilon}_{1}<\epsilon$ we conclude that $\vec{K} \in B(K, \epsilon)$ and then

$$
B=B\left(K_{1}, \epsilon_{1}\right) \cap B\left(K_{2}, \epsilon_{2}\right)=\bigcup_{\tilde{K} \in B} B(\tilde{K}, \epsilon(\tilde{K}))
$$

so, $\mathcal{B}=\{B(K, \epsilon): K \in \mathcal{K}$ and $\epsilon>0\}$ is a basis for a topology $\Gamma$ over $\mathcal{K}$.

Proposition 1. The topology $\Gamma$ is induced by the metric $\rho: \mathcal{K} \times \mathcal{K} \longrightarrow[0, \infty]$ defined by:

$$
\rho(K, \tilde{K})=\sup \{\epsilon \geq 0: \tilde{K} \notin B(K, \epsilon)\} .
$$

Proof: First we will show that $\rho$ is a metric. Suppose that $\rho(K, \tilde{K})=0$, then there is a sequence $\epsilon_{k} \downarrow 0$ such that $\tilde{K} \in B\left(K, \epsilon_{k}\right), k \in \mathbb{N}$, so for every $k \in \mathbb{N}$ there is an $\tilde{\epsilon}_{k}$ such that $0<\tilde{\epsilon}_{k}<\epsilon_{k}$ and for all $n \in \mathbb{N}$ :

$$
2^{-\tilde{\epsilon}_{k} n}<\frac{\ell\left(I_{n}\right)}{\ell\left(\tilde{I}_{n}\right)}<2^{\tilde{\epsilon}_{k} n}
$$

Consequently for every $n \in \mathbb{N}$ we conclude, letting $k$ tends to $\infty$, that $\frac{\ell\left(I_{n}\right)}{\ell\left(\tilde{I}_{n}\right)}=1$, but since $K$ and $\tilde{K}$ are central Cantor sets we see that $K=\tilde{K}$. Conversely it is clear that if $K=\tilde{K}$, then $\rho(K, \tilde{K})=0$. From definition of $B(K, \epsilon)$ it is immediate that $\tilde{K} \in B(K, \epsilon)$, if and only if, $K \in B(\tilde{K}, \epsilon)$ and then simmetry of $\rho$ follows. To show the triangular inequality, consider $K, \widetilde{K}$ and $\bar{K} \in \mathcal{K}$, and let first suppose that $\rho(K, \bar{K})$ and $\rho(\bar{K}, \tilde{K})$ are finite. Let $\epsilon_{1}$ and $\epsilon_{2}$ be positive constants 
such that $\rho(K, \bar{K})<\epsilon_{1}$ and $\rho(\tilde{K}, \tilde{K})<\epsilon_{2}$, then $\bar{K} \in B\left(K, \epsilon_{1}\right)$ and $\tilde{K} \in B\left(K, \epsilon_{2}\right)$. From the definition of $\rho$ there are positive constants $\bar{\epsilon}_{1}$ and $\tilde{\epsilon}_{2}$ such that

$$
0<\rho(K, \bar{K})<\bar{\epsilon}_{1}<\epsilon_{1} \quad \text { and } \quad 2^{-\bar{\epsilon}_{1} n}<\frac{\ell\left(I_{n}\right)}{\ell\left(\bar{I}_{n}\right)}<2^{\bar{\tau}_{1} n}
$$

and

$$
0<\rho(\bar{K}, \tilde{K})<\tilde{\epsilon}_{2}<\epsilon_{2} \text { and } 2^{-\tilde{\epsilon}_{2} n}<\frac{\ell\left(\bar{I}_{n}\right)}{\ell\left(\tilde{I}_{n}\right)}<2^{\bar{\epsilon}_{2} n} .
$$

Combinig the two last inequalities we obtain

$$
2^{-\left(\bar{\epsilon}_{1}+\bar{\epsilon}_{2}\right) n}<\frac{\ell\left(I_{n}\right)}{\left.\ell \tilde{I}_{n}\right)}<2^{\left(\bar{\epsilon}_{1}+\tilde{\epsilon}_{2}\right) n}
$$

consequently $\tilde{K} \in B\left(K, \bar{\epsilon}_{1}+\tilde{\epsilon}_{2}+\epsilon\right)$ for all $\epsilon>0$ and then $\rho(K, \tilde{K})<\bar{\epsilon}_{1}+\tilde{\epsilon}_{2}+\epsilon$. Since $\epsilon$ is arbitrary, we obtain $\rho(K, \tilde{K}) \leq \bar{\epsilon}_{1}+\tilde{\epsilon}_{2}$. Letting $\epsilon_{1} \downarrow \rho(K, \bar{K})$ and $\epsilon_{2} \downarrow \rho(\bar{K}, \tilde{K})$ we conclude that $\rho(K, \tilde{K}) \leq \rho(K, \bar{K})+\rho(\bar{K}, \tilde{K})$. Finally let suppose that $d(K, \tilde{K})=\infty$. The argument above shows that if $d(K, \bar{K})$ and $d(\bar{K}, \tilde{K})$ are both finite then $d(K, \tilde{K})$ is finite. Therefore under our suposition we do have that al least one of the $d(K, \bar{K})$ or $d(\bar{K}, \tilde{K})$ must be infinite. metric.

This proves the triangular inequality and then we conclude that $\rho$ is a

Now we show that the topology $\Gamma$ is induced by the metric $\rho$. In fact, $\rho(K, \tilde{K})<\epsilon$, if and only if, there is $\epsilon_{1}<\epsilon$ such that $\tilde{K} \in B\left(K, \epsilon_{1}\right)$, if and only if, there is $0<\tilde{\epsilon}<\epsilon$ such that $2^{-\tilde{\epsilon} n}<\frac{\ell\left(I_{n}\right)}{\ell\left(\tilde{I}_{n}\right)}<2^{\tilde{c} n}$, if and only if $\tilde{K} \in B(K, \epsilon)$, and then we conclude that

$$
B(K, \epsilon)=\{\tilde{K}: \rho(K, \tilde{K})<\epsilon\}
$$

and this finishes the proof.

\section{Continuity of limit capacity and Hausdorff dimension} lemma.

In order to prove the continuity of limit capacity we do need the next

Lemma 1. For $\eta \in S^{+}$let $\epsilon_{n}=2^{-n}\left(\sum_{i=n+1}^{\infty} \eta_{i}\right)$, then the limit capacity of $K(\eta)=\Pi_{n \in \boldsymbol{N}} K_{n}$ is given by $d(K(\eta))=\limsup _{n \rightarrow \infty} \frac{\ln 2^{n}}{-\ln \varepsilon_{n}}$. 
Proof: Given $\epsilon>0$ there exists $m \in \mathbb{N}$ such that $\epsilon_{m+1} \leq \epsilon \leq \epsilon_{m}$ and then

$$
n\left(\epsilon_{m}, K\right)=2^{m}<n(\epsilon, K) \leq 2^{m+1}=n\left(\epsilon_{m+1}, K\right),
$$

consequently

$$
2^{-1} n\left(\epsilon_{m+1}, K\right)<n(\epsilon, K) \leq 2 n\left(\epsilon_{m}, K\right) .
$$

Hence,

$$
\ln n\left(\epsilon_{m+1}, K\right)-\ln 2<\ln n(\epsilon, K) \leq \ln n\left(\epsilon_{m}, K\right)+\ln 2 .
$$

By the other hand,

$$
\frac{1}{-\ln \epsilon_{m+1}} \leq \frac{1}{-\ln \epsilon}<\frac{1}{-\ln \epsilon_{m}}
$$

and combining the last two inequalities we obtain

$$
\frac{\ln n\left(\epsilon_{m+1}, K\right)}{-\ln \epsilon_{m+1}}+\frac{\ln 2}{\ln \epsilon_{m+1}} \leq \frac{\ln n(\epsilon, K)}{-\ln \epsilon} \leq \frac{\ln n\left(\epsilon_{m}, K\right)}{-\ln \epsilon_{m}}-\frac{\ln 2}{\ln \epsilon_{m}}
$$

Since $\frac{\ln 2}{\ln \epsilon_{m}} \longrightarrow 0$ as $m \longrightarrow \infty$, we conclude

$$
d(K)=\limsup _{\epsilon \rightarrow 0} \frac{\ln n(\epsilon, K)}{-\ln \epsilon}=\limsup _{n} \frac{\ln 2^{n}}{-\ln \epsilon_{n}} .
$$

Remark 1. In [2] it is shown that the Hausdorff dimension of elements in $\mathcal{K}$ coincide with $\liminf \epsilon_{\epsilon \rightarrow 0} \frac{\ln n(\epsilon, K)}{-\ln \epsilon}$ and then the argument above applies to prove that

$$
H D(K)=\limsup _{n \rightarrow \infty} \frac{\ln 2^{n}}{-\ln \epsilon^{n}} .
$$

Proposition 2. The Hausdorff dimension and the limit capacity of $K=K(\eta)$ in $\mathcal{K}, \mathcal{K}$ endowed with the topology $\Gamma$ depend continously o $K$.

Proof: Let $\epsilon>0$ and let $\tilde{K} \in B(K, \epsilon)$. Then there is $\tilde{\epsilon}<\epsilon$ such that for all $n \in \mathbb{N}$

$$
2^{-\bar{\epsilon} n}<\frac{\ell\left(I_{n}\right)}{\ell\left(\tilde{I}_{n}\right)}<2^{\tilde{\epsilon} n}
$$

Taking natural logarithm,

$$
\ln \left(\ell\left(\tilde{I}_{n}\right)\right)-\tilde{\epsilon} \ln 2^{n}<\ln \left(\ell\left(I_{n}\right)\right)<\ln \left(\ell\left(\tilde{I}_{n}\right)\right)+\tilde{\epsilon} \ln 2^{n},
$$


and multiplying by the inverse of $-\ln 2^{n}$ yields

$$
\frac{1}{\frac{\ln 2^{n}}{-\ln \left(\ell\left(\tilde{I}_{n}\right)\right)}}+\tilde{\epsilon}>\frac{-\ln \left(\ell\left(I_{n}\right)\right)}{\ln 2^{n}}>\frac{1}{\frac{\ln 2^{n}}{-\ln \left(\ell\left(\tilde{I}_{n}\right)\right)}}-\tilde{\epsilon}
$$

Since $0<\ell\left(I_{n}\right), \ell\left(\tilde{I}_{n}\right)<2^{-n}<1$, we have

$$
0<\frac{\ln 2^{n}}{-\ln \left(\ell\left(I_{n}\right)\right)}<1 \text { and } 0<\frac{\ln 2^{n}}{-\ln \left(\ell\left(\tilde{I}_{n}\right)\right)}<1
$$

and henceforth we conclude

$$
\left|\frac{\ln 2^{n}}{-\ln \left(\ell\left(I_{n}\right)\right)}-\frac{\ln 2^{n}}{-\ln \left(\ell\left(\tilde{I}_{n}\right)\right)}\right| \leq\left|\frac{1}{\frac{\ln 2^{n}}{-\ln \left(\ell\left(I_{n}\right)\right)}}-\frac{1}{\frac{\ln 2^{n}}{-\ln \left(\ell\left(\tilde{I}_{n}\right)\right)}}\right|<\tilde{\epsilon}<\epsilon .
$$

Therefore, continuity of Hausdorff dimension and limit capacity follow, from this inequality, lemma 1 and remark 1, and this finishes the proof.

Remark 2. If $\eta:[0,1] \longrightarrow S^{+}, \eta(t)=\left\{\eta_{n}(t)\right\}_{n=1}^{\infty}$ satisfies $2^{-\left|t_{1}-t_{2}\right| n}<$ $\frac{1-\sum_{i=1}^{n} \eta_{1}\left(t_{1}\right)}{1-\sum_{t=1}^{n} \eta_{i}\left(t_{2}\right)}<2^{\left|t_{1}-t_{2}\right| n}$ for all $n \in \mathbb{N}$ and $t_{1}, t_{2} \in[0,1]$, then the arc $\alpha:[0,1] \longrightarrow$ $\mathcal{K}, \alpha(t)=K(\eta(t))$, with $\eta(t)=\left\{\eta_{n}(t)\right\}_{n \in N}$ is uniformiy continuous. In fact, let $t_{0} \in[0,1]$ and let $\epsilon>0$, choose $\tilde{\epsilon}$ such that $\frac{\epsilon}{2}<\tilde{\epsilon}<\epsilon$. If $\delta=\frac{\epsilon}{2}$ and $\left|t_{0}-t\right|<\delta$, then $-\left|t_{0}-t\right|>-\tilde{\epsilon}$ and for all $n \in \mathbb{N}$.

$$
2^{-\tilde{c} n}<2^{-\left|t_{0}-t\right| n}<\frac{\ell\left(I_{n}\left(t_{0}\right)\right)}{\ell\left(I_{n}(t)\right)}<2^{\left|t_{0}-t\right| n}<2^{\tilde{e} n}
$$

i.e., $\alpha(t) \in B\left(\alpha\left(t_{0}\right), \epsilon\right)$ and the $\operatorname{arc} \alpha:[0,1] \longrightarrow \mathcal{K}$ is uniformly continuous.

As an example, we show that given a central Cantor set $K$, it is possible to construct a non trivial $\operatorname{arc} \alpha:[0,1] \longrightarrow \mathcal{K}$ with constant capacity such that $K=\alpha(0)$. $n \in \mathbb{N}$

If we define $\eta_{i}:[0,1] \longrightarrow[0,1]$ such that $K=K\left(\left\{\eta_{i}(0)\right\}_{i=1}^{\infty}\right)$ and for all

$$
2^{-\left|t_{1}-t_{2}\right| n}<\frac{1-\sum_{i=1}^{n} \eta_{i}\left(t_{1}\right)}{1-\sum_{i=1}^{n} \eta_{i}\left(t_{2}\right)}<2^{\left|t_{1}-t_{2}\right| n}
$$


then it is easy to see (the same argument as above), that $d\left(K\left(\left\{\eta_{i}(t)\right\}_{i=1}^{\infty}\right)\right)=$ $d\left(K\left(\left\{\eta_{i}(s)\right\}_{i=1}^{\infty}\right)\right)$ and the limit capacity well be constant along the arc. To define $\eta_{i}:[0,1] \longrightarrow[0,1]$ with the required properties, we let formally consider $f_{n}(t)=\ln \left(\sum_{i=n+1}^{\infty} \eta_{i}(t)\right)$. Taking natural logarithm in the last inequality, we obtain

$$
-\ln 2^{n}<\frac{f_{n}\left(t_{1}\right)-f_{n}\left(t_{2}\right)}{\left|t_{1}-t_{2}\right|}<\ln 2^{n}
$$

This suggest us to define $f_{n}$ differentiable and such that for all $n \in \mathbb{N}$

$$
-\ln 2<\frac{d f_{n}}{d t}(t)<\ln 2
$$

Let us define $\eta_{i}(0)$ such that $K=K\left(\left\{\eta_{i}(0)\right\}_{i=1}^{\infty}\right)$ and let consider $f_{n}(t)=$ $a_{n} t+b_{n}$, where $b_{n}=f_{n}(0)=\ln \left(\sum_{i=n+1}^{\infty} \eta_{i}(0)\right), a_{n} \downarrow 0$, and $a_{n}<\min \left\{-b_{n}, \ln 2\right\}$. Now, we define $\eta_{1}=1-e^{f_{1}(t)}$ and inductively $\sum_{i=1}^{n} \eta_{i}(t)=1-e^{f_{n}(t)}$. In this way, $\eta_{i}:[0,1] \longrightarrow[0,1]$ is of class $C^{\infty}$ for all $i \in \mathbb{N}$. Since $b_{n} \longrightarrow-\infty$ and $a_{n} \longrightarrow 0$, we have

$$
\sum_{i=1}^{\infty} \eta_{i}(t)=\lim _{n \rightarrow \infty} \sum_{i=1}^{n} \eta_{i}(t)=\lim _{n \rightarrow \infty}\left(1-e^{\rho_{n}(t)}\right)=1
$$

i.e., $\alpha(\eta(t))=K\left(\left\{\eta_{i}(t)\right\}_{i=1}^{\infty}\right) \in \mathcal{K}$. Since $0<a_{n}<\ln 2$ for all $n \in \mathbb{N}$, we have

$$
-\ln 2<\frac{d}{d t} f_{n}(t)=a_{n}<\ln 2,
$$

and then the limit capacity is constant along the arc. Continuity of the $\operatorname{arc} \alpha$ follows from the next inequality:

$$
2^{-\left|t_{1}-t_{2}\right| n}<2^{-\left|t_{1}-t_{2}\right|}<\frac{1-\sum_{i=1}^{n} \eta_{i}\left(t_{1}\right)}{1-\sum_{i=1}^{n} \eta_{i}\left(t_{2}\right)}<2^{\left|t_{1}-t_{3}\right|}<2^{\left|t_{1}-t_{2}\right| n}
$$

\section{REFERENCES}

[1] Hocking, J. \& Young, G.: Topology. Addison-Wesley Series in Mathematics., 1961. 
[2] Tricot, C.: Two definitions of fractional dimensions. Mathematical Proceedings of the Cambridge Philosophical Society. 91, 1982, pp 57-74.

Recibido : 30 de Septiembre de 1992.

Eduardo Muñoz M. and Jaime Vera

Departamento de Matemática

Universidad Católica del Norte

Casilla 1280 Antofagasta, Chile. 